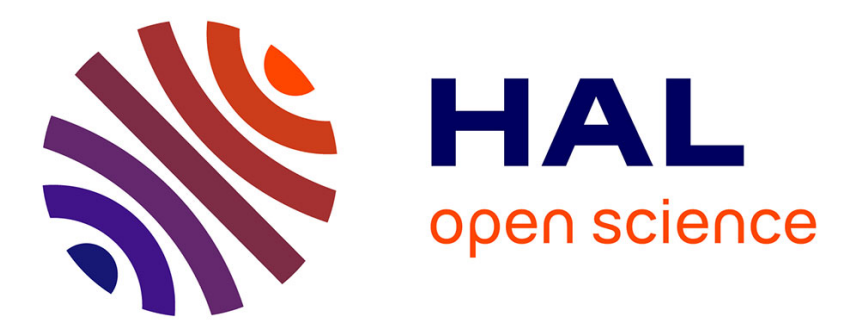

\title{
Environmental and individual characteristics associated with depressive disorders and mental healthcare use
}

\author{
Margot Annequin, Alain Weill, Frédérique Thomas, Basile Chaix
}

\section{To cite this version:}

Margot Annequin, Alain Weill, Frédérique Thomas, Basile Chaix. Environmental and individual characteristics associated with depressive disorders and mental healthcare use. Annals of Epidemiology, 2015, 25 (8), pp.605-612. 10.1016/j.annepidem.2015.02.002 . hal-01118138

\section{HAL Id: hal-01118138 \\ https://hal.sorbonne-universite.fr/hal-01118138}

Submitted on 18 Feb 2015

HAL is a multi-disciplinary open access archive for the deposit and dissemination of scientific research documents, whether they are published or not. The documents may come from teaching and research institutions in France or abroad, or from public or private research centers.
L'archive ouverte pluridisciplinaire HAL, est destinée au dépôt et à la diffusion de documents scientifiques de niveau recherche, publiés ou non, émanant des établissements d'enseignement et de recherche français ou étrangers, des laboratoires publics ou privés. 


\section{Environmental and individual characteristics associated with depressive disorders and} mental healthcare use

Running title: Environment, depression, healthcare use

Margot Annequin MPH ${ }^{\mathrm{a}, \mathrm{b}}$, Alain Weill MD, $\mathrm{PhD}^{\mathrm{c}}$, Frédérique Thomas $\mathrm{PhD}^{\mathrm{d}}$, Basile Chaix $\mathrm{PhD}^{\mathrm{a}, \mathrm{b}, *}$

${ }^{a}$ INSERM, UMR_S 1136, Pierre Louis Institute of Epidemiology and Public Health, Department of social epidemiology, 75012, Paris, France

${ }^{b}$ Sorbonne Universités, UPMC Univ Paris 06, UMR_S 1136, Pierre Louis Institute of Epidemiology and Public Health, Department of social epidemiology, 75012, Paris, France

${ }^{c}$ Caisse Nationale d'Assurance Maladie des Travailleurs Salariés, 75020 Paris, France

${ }^{d}$ Centre d'Investigations Préventives et Cliniques, 75116 Paris, France

* Corresponding author. INSERM, UMR_S 1136, Pierre Louis Institute of Epidemiology and Public Health, Department of social epidemiology, 75012, Paris, France. Tel: +331 447386 64; fax: +33144738462.

E-mail address: basile.chaix@upmc.fr (B. Chaix).

Abstract word count: 231

Word count (excluding title page, abstract, references, figures, and tables): 4053

Number of tables: 5 


\begin{abstract}
Purpose: Few studies examined the relationship between neighborhood characteristics and both depressive disorders and the corresponding mental healthcare utilization. The aim of our study was to investigate neighborhood effects on depressive symptomatology, antidepressant consumption, and the consultation of psychiatrists.
\end{abstract}

Methods: Data from the French RECORD Study ( $\mathrm{n}=7290,2007-2008$, age 30-79 years) were analyzed. Depressive symptomatology was cross-sectionally assessed with the QD2A scale. Healthcare reimbursement data allowed us to assess antidepressant consumption and psychiatric consultation prospectively over 18 months. Multilevel logistic regression models were estimated. Results: The risk of depressive symptoms increased with decreasing personal educational level and unemployment, and slightly with decreasing neighborhood income. In a sample comprising participants with and without depressive symptoms, high individual and parental educational levels were both associated with the consultation of psychiatrists. In this sample, a low personal educational level increased the odds of consumption of antidepressants. No heterogeneity between neighborhoods was found for antidepressant consumption. However, the odds of consulting psychiatrists increased with median neighborhood income and with the density of psychiatrists, after adjustment for individual characteristics. Among depressive participants only, a particularly strong gradient in the consultation of psychiatrists was documented according to individual socioeconomic status.

Conclusion: Future research on the relationships between the environments and depression should take into account healthcare utilization related to depression and consider the spatial accessibility to mental health services among other environmental factors. 
Keywords: depression, socioeconomic factors, residence characteristics, healthcare disparities, mental health 


\section{List of abbreviations and acronyms}

ESEMeD, European Study of the Epidemiology of Mental Disorders

IRIS, Îlots regroupés pour l'information statistique

RECORD, Residential Environment and CORonary heart Disease

QD2A, Questionnaire D’auto-évaluation de la Dépression, abrégé

TRIRIS, Trois IRIS 


\section{Introduction}

A study found that in the general French population $6.7 \%$ of people suffered from a depressive disorder over the 12 previous months [1]. Studies have shown how individual sociodemographic factors are associated with depression and use of mental health services [1-3]. More recently, studies have investigated whether features of neighborhoods such as the socioeconomic context, the physical environment, and social interactions (violence, disorders, etc.) were associated with the odds of depressive disorders [4, 5]. Despite some inconsistent results between studies, the evidence suggests that neighborhood deprivation plays a role in the development of depressive symptoms. Features of the neighborhood may operate as stressors or buffers of individual-based sources of stress related to mental illness [4].

Despite universal coverage in France, a study found that, among individuals with mental disorders, only $25.7 \%$ reported using formal health services for this condition [6]. Unequal use of mental health care has been documented in France. Socioeconomic status and other individual factors (low perceived need, lack of mental health literacy, etc.) were identified as determinants of mental health care use [7-10]. Organizational factors, such as the rate of reimbursement of psychotherapies or gatekeeping policies, were also found to influence mental health service utilization $[11,12]$. However, although there is an unequal geographical distribution of mental health professionals (the department of Paris had a density of 72.8 psychiatrists for 100000 inhabitants in 2014, as compared to 17.5 in the poorer, immediately adjacent county of Seine Saint Denis[13]), much less is known about environmental influences on mental health services utilization $[9,14,15]$.

Overall, as a background of the present study on individual/neighborhood determinants of depressive symptoms and related health care use, the French situation is characterized by a low prevalence of mental health service use in the depressive population, an inappropriate use of 
care, and an overconsumption of antidepressants in the French general population [16-18], and by an unequal distribution of mental health professionals [13].

\section{Objectives}

In order to gather information on depressive symptoms, patterns of use of mental health service, and potential underuse of care in the segment of the population requiring care, our overall objectives were to assess the individual and neighborhood determinants of depressive symptoms and of the use of two corresponding mental healthcare services (antidepressant consumption and consultation of a psychiatrist).

Given that consulting a psychiatrist is not restricted to people with depressive disorders and considering the excessive consumption of antidepressants in France even among people without depression, the present article first investigates the determinants of mental healthcare use in the whole study population (including depressive and non-depressive participants). Moreover, to identify potential situations of underuse of care, we also explored comparatively the patterns of use of services among participants with depressive symptoms.

Our specific aims were to evaluate the between-neighborhood variability in the three outcomes examined; to assess whether socioeconomic and social features of residential neighborhoods (i.e., related to patterns of social interactions) were associated with depression and healthcare use; and to investigate whether the spatial accessibility to healthcare services influenced the healthcare use of the participants. 


\section{Methods}

\section{Population}

We used data from the first wave of the RECORD Cohort Study (www.record-study.org) that includes 7290 participants who were recruited between March 2007 and February 2008 [19-24]. The participants benefitted from a free medical checkup, offered every 5 years by the French National Health Insurance System for Salaried Workers to all working and retired employees and their families. Participants were recruited without a priori sampling during these 2-hour-long preventive checkups conducted by the Centre d'Investigations Préventives et Cliniques. Eligibility criteria were age 30 to 79 years, ability to fill out the study questionnaires, and residence in 1 of 10 (out of 20) administrative divisions of Paris or 111 other municipalities of the metropolitan area selected a priori. Among the participants, $83.6 \%$ accepted to participate and completed the data collection protocol. All participants were geocoded with accuracy based on their residential address in 2007-2008. All participants signed an informed consent form. The study protocol was approved by the French Data Protection Authority.

Sixty-four participants were removed from the analyses of healthcare use because they could not be linked to the National Health Insurance Fund database on healthcare use. Our study population includes 7290 participants for the analyses on depression and 7226 for those on the consumption of antidepressants and psychiatric consultations.

\section{Measures}

Depressive symptomatology

The presence of a depressive symptomatology was assessed with the QD2A depression scale from Pichot [25]. This scale is based on 13 dichotomous binary items (true or false). A score above 7 was coded as a depressive symptomatology. 
Healthcare use

Data from the French National Health Insurance Fund were merged at the individual level to the RECORD Study database. We created a variable on the reimbursement of antidepressants over the 18 months after the inclusion in the study. We used the code N06A from the Anatomical Therapeutic Chemical system to identify the consumption of antidepressants. Differences in the frequency of consumption or in the therapeutic agent were not taken into account.

Based on reimbursement data, a binary variable indicated whether the participants had seen a private psychiatrist over the 18 months after baseline (ambulatory consultations). We could take into account neither the consultations of psychologists (not reimbursed by the healthcare system) nor the ambulatory psychiatric consultations made in public structures (not available in the reimbursement database). Inpatient hospital treatment for depression was not considered, as beyond the scope of the present study.

Individual covariates

The following socio-demographic characteristics reported by the participants were considered: age, sex, marital status, personal education, parental education, occupation, employment status, household income, financial strain, country of birth, and Health insurance situation.

Neighborhood-level characteristics

The following socioeconomic variables were defined at the neighborhood level using databases geocoded at the building level: (1) the proportion of residents aged $\geq 15$ years with an 
upper tertiary education (2006 census); and (2) median income in 2006 (Tax Registry of General Directorate of Taxation).

Regarding the spatial accessibility to care supply, we took into account the density per $\mathrm{km}^{2}$ of private psychiatrists and the density per $\mathrm{km}^{2}$ of private general practitioners. We also determined the overall density of physicians (taking into account general practitioners and all medical specialties) and the total number of all types of services in the neighborhood (shops, leisure facilities, administrative services, etc.).

All these socio-demographic and service variables were defined in buffer areas that were centered on the residence and took into account the street network, using the ArcGIS 10.0 Network Analyst. Different variables were defined, with a $500 \mathrm{~m}, 1 \mathrm{~km}$, or $2 \mathrm{~km}$ radius along the street network.

Ecometric variables on neighborhood social interactions were created with 3-level multilevel models (items, individuals, neighborhoods) applied to environmental survey items collected during the survey [19]. Multilevel models allowed us to aggregate at the individual level the information provided by each respondent on each dimension and to combine the answers of the different individuals of the same neighborhood to construct indicators at the neighborhood level. Responses to questionnaire items for each dimension were aggregated to construct variables on the following dimensions: neighborhood social cohesion (4 items: neighbors helpful to each other, collective neighborhood identity, collective action to solve problems, close-knit neighborhood); stressful social interactions among neighbors (5 items : victim or witness of theft/assault, incivility, feeling of insecurity, friendly/polite neighbors, noisy neighbors); neighborhood mistrust and hostility (5 items: mistrust towards neighbors, avoid neighbors, angry against neighbors, friendly/polite neighbors, do not appreciate certain inhabitants); and stigmatized neighborhood identity ( 2 items: poor neighborhood reputation, ashamed of one's 
neighborhood). All neighborhood variables were divided into 4 categories comprising a similar number of individuals.

\section{Statistical Analysis}

Multilevel logistic models were estimated to take into account the correlation in the odds of depressive symptoms or healthcare utilization within residential areas [26]. We compared different geographical levels (IRIS, TRIRIS, and municipalities) in the multilevel analyses to identify the scale enabling us to capture the existing spatial variations with the available statistical power (Table 1). We used the median odds ratio to quantify the heterogeneity in the outcomes between neighborhoods. As previously explained [27], the median odds ratio quantifies on the odds ratio scale the increase in the odds of the outcome between the area at lowest risk and the area at highest risk when randomly selecting individuals from two different areas.

We first estimated models adjusted for age and gender. Second, we parsimoniously selected the individual variables to introduce in the models (variables not associated with the outcomes were removed). Third, the contextual variables were further introduced into the models one by one and simultaneously when they were independently associated with the outcome. To assess whether the relationship between the spatial accessibility to psychiatrists and the consultation of psychiatrists was specifically attributable to the density of psychiatrists, the model was further adjusted for the overall density of physicians and the number of services in the neighborhood. Sensitivity analyses on the neighborhood variables were conducted comparing street network buffers with a radius of $500 \mathrm{~m}, 1 \mathrm{~km}$, or $2 \mathrm{~km}$. As only minor differences were observed, the analyses below rely on $1 \mathrm{~km}$ radius street network buffers. 
Finally, to identify potential situations of underuse of services, we tabulated the rate of consumption of antidepressants and consultation of psychiatrists according to the main individual/neighborhood variable among participants with depressive symptoms. The reduced sample of depressive participants did not allow us to perform a formal multivariable analysis in this subpopulation

\section{Results}

Overall, 7.8\% of the participants had a QD2A score above 7 (suggestive of depressive symptoms). In the sample of 7226 participants, $9.9 \%$ were reimbursed for antidepressants over 18 months after recruitment, and $4.7 \%$ consulted a psychiatrist over the same period. Among participants with depressive symptoms $(n=566)$, these figures were respectively $35.7 \%$ and $12.0 \%$.

\section{Variations between residential areas}

Models in Table 1 present the magnitude of the between-area heterogeneity for the 3 outcomes. As expected, the magnitude of variations between areas tended to be larger for all 3 outcomes when smaller areas (IRIS neighborhoods) were considered. As there was sufficient power to detect variations in the odds of depressive symptoms and psychiatrist consultation between IRIS neighborhoods, such area level was selected for the subsequent multilevel analyses. Multilevel logistic models adjusted for age and gender indicated that the odds of consulting a psychiatrist were markedly different according to the participant's neighborhood. The heterogeneity between neighborhoods in the odds of being depressive was of smaller magnitude. On the opposite, the odds of consuming antidepressants were relatively homogeneous across neighborhoods. 
Individual correlates of depressive symptoms and mental healthcare use

Depressive symptoms

As shown in Table 2, after mutual adjustment for individual variables, the risk of depressive symptoms increased with decreasing personal educational level, unemployment and retirement, and being alone. Income was not associated with depressive symptomatology, but reporting financial strain was related to much higher odds of it.

The random effect variance at the IRIS level indicates that there was no variability between neighborhoods in the odds of depressive symptoms after taking individual characteristics into account.

Healthcare utilization

After adjustment for depressive status, comparable individual characteristics were associated with the consumption of antidepressants and with the visit to a psychiatrist, but for some of these explanatory variables in the opposite direction for the two outcomes (Table 3).

Gender, country of birth, and marital status were associated with mental healthcare utilization in the same way for the 2 outcomes: women, people born in France, and people living alone had higher odds to either consume antidepressants or consult a psychiatrist.

Regarding education, individual and parental educational levels were both positively associated with the consultation of psychiatrists (independent associations). Differently, individual and parental educational levels were associated in the opposite direction with the consumption of antidepressants: a low parental educational level was associated with lower odds of consumption of antidepressants while a low personal educational level increased the odds of consumption. 
Financial strain was no longer associated with healthcare utilization behaviors after adjustment, but participants with a low income level had higher odds to consult a psychiatrist after adjustment for the other factors.

The multilevel model did not detect any residual variability between neighborhoods in the odds of antidepressant consumption. The odds of consultation of psychiatrists were found to vary between neighborhoods even after adjustment for individual demographic and socioeconomic factors (bottom of Table 3).

\section{Environmental correlates of depressive symptoms and mental healthcare use}

Depressive symptoms

Table 2 shows that despite the absence of heterogeneity between neighborhoods suggested by the residual between-neighborhood variance, independently from individual characteristics, the median income of the neighborhood was associated with the odds of depressive symptoms, with a weaker risk of depression in richer neighborhoods.

\section{Healthcare utilization}

No association was found between the socioeconomic or social interactional characteristics of residential environments or the spatial accessibility to healthcare services and the consumption of antidepressants.

As shown in Table 4, after adjustment for individual socioeconomic variables, the odds of consulting psychiatrists increased with median neighborhood income assessed within $1 \mathrm{~km}$ from the residence. Such odds of consulting also increased with the density of psychiatrists in the neighborhood. When the two contextual variables were introduced simultaneously into the model, only the density of psychiatrists remained associated with the odds of consulting, even if 
such odds also tended to increase slightly with increasing median neighborhood income. Even if it is not possible to compare the variance of successive logistic models [28, 29], Table 4 suggests that including the density of psychiatrists in the model led to a larger decrease of the betweenneighborhood variance than including neighborhood income.

To examine whether the association identified between the density of psychiatrists and the consultation of psychiatrists is likely to be attributable to the density of psychiatrists itself, we reestimated the model with additional adjustment either for the overall density of physicians or for the overall density of services. In these models, only the density of psychiatrists was related to the consultation of psychiatrists, which suggests that the association observed may be attributable to a specific link between the density of psychiatrists and the use of the corresponding services. Neighborhood social interactions were not associated with the consultation of psychiatrists.

\section{Individual correlate of mental health care use in the depressive population}

Table 5 compares demographic and socioeconomic gradients in the consumption of antidepressants and in psychiatric consultations between the total sample (comprising participants with and without depressive symptoms) and the sample of depressive participants. This comparison suggests that the decreased use of antidepressants among high education and high income participants that was observed in the whole population was not documented among depressive participants. On the opposite, the socioeconomic gradient in the consultation of private psychiatrists was found to be much stronger in the depressive population (with healthcare needs) than in the whole sample. 


\section{Discussion}

Based on individual and neighborhood socioeconomic variables, we found that there were disparities in the prevalence of depressive symptoms and its treatment between socially advantaged and disadvantaged groups. A high individual education level and a high parental education level had a cumulative effect on the odds of psychiatric consultation. Socioeconomic disparities in the use of psychiatric consultations were much stronger among depressive participants than in the overall sample. Regarding neighborhood influences, a weak neighborhood income effect was documented on the odds of depression. While no area heterogeneity was found for the consumption of antidepressants, the odds of psychiatric consultation increased with the density of psychiatrists in the residential neighborhood, after adjustment for individual and neighborhood characteristics.

\section{Strengths and limitations}

The first strength of this analysis is that we used objectively-assessed rather than self-reported mental healthcare use indicators, which allowed us to minimize memory/information biases. While studies based on administrative healthcare use data often lack socioeconomic information, merging administrative reimbursement data to the RECORD Study allowed us to adjust the associations of interest for various socio-demographic covariates and for the depression status.

A second methodological strength of the work is that we took into account the street network to determine the spatial accessibility to healthcare services, and used multilevel models to link environmental factors to health status and healthcare use after adjustment for individual factors. Third, we used different measures of individual socioeconomic status (individual and parental education level, income, self-perceived financial strain), allowing us to disentangle the influence of the cultural and financial dimensions of socioeconomic status on healthcare utilization. 
However the main limitation of the present study is our sample which does not include individuals aged between 18 and 30 years old, is restricted to the Paris metropolitan area, and recruited people attending preventive healthcare examinations without any a priori randomization (convenience sample). A higher rate of participation to the study was observed among men, highly educated individuals, and among people residing close to the health centers participating in the recruitment and in neighborhoods with high income, high property values, high proportion of the population looking for work, and low built surface and low building height [30].

Other limitations include the fact that we considered private psychiatrists but neither public psychiatrists in health centers nor psychologists (see implications below); the limited size of the sample of depressive participants; and the fact that considering the reimbursement of a drug as an indicator of consumption has its limitations (a participant buying a drug may not consume it).

\section{Socio-demographic determinants of depressive symptoms}

Although the RECORD sample is not representative of the whole French population, our results confirm the findings of previous studies : women [31, 32], young people [33], and participants with a low socioeconomic status [2] had a higher prevalence of depressive symptoms.

\section{Individual socio-demographic determinants of mental healthcare use}

In France, individuals facing depressive symptoms can rely on various professionals: general practitioners, psychiatrists, psychologists, or other mental health professionals [11]. General practitioners are usually the first professionals that people are consulting when facing depression [8], and they are the first prescriber of antidepressants. Then, when referring their patients in 
need of mental healthcare to health professionals, general practitioners primarily send them to psychiatrists [12].

The relationship documented in our study suggesting that low educated participants had higher odds to consume antidepressants was expected from previous research [34-36]. However, it is difficult to explain at this stage of the research why parental education was associated in the opposite direction with antidepressant consumption. Our finding that the educational gradient in the consumption of antidepressants disappeared among participants with depressive symptoms further supports the idea that the excess use of antidepressants by low educated people in the overall sample may be to some extent inappropriate. As regard to consultation with a psychiatrist: women, people living alone, and participants born in the country had higher odds to seek help from a psychiatrist $[6,9]$.

Our study also brings new information on the relationship between socioeconomic status and the consultation of psychiatrists. It suggests that individual and parental educational levels had a cumulative effect on the odds of psychiatric consultation. These results are coherent with previous studies that emphasize that socially advantaged populations more frequently rely on specialized care $[9,34,37,38]$. Our study shows the benefits of considering both the educational and financial dimensions of socioeconomic status, which can influence healthcare use in a different or even opposite way (as found in the adjusted model for psychiatric consultations in the overall sample). A notable finding is that the socioeconomic gradient in the consultation of psychiatrists (higher status, higher use) was much stronger among participants needing care (those with depressive symptoms) than in the overall population, and that both education and income may have contributed to the gradient among depressive participants. If not attributable to the fact that public psychiatrists were not accounted for in this study, these results may be concern for equity in the access to care. 
It would have been interesting to take into consideration the consultations of psychologists and the ambulatory consultations of psychiatrists made at the hospital or in public mental healthcare centers. Currently, no data are available in France in the general population on the use of psychologists as these consultations are not reimbursed by the national health insurance system. In France, private psychiatrists are to a large extent reimbursed by the healthcare system, while consultations of psychologists are out-of-pocket costs for the patients that are reimbursed only very rarely by very particular insurance schemes. Regarding public psychiatrists available in specific public centers, the service is free but long waiting delays can be expected before accessing to a physician [9]. Overall, socioeconomic inequalities in the access to healthcare may be intermediary for the service that was considered in the present study (private psychiatrists). While larger socioeconomic inequalities may be expected for the access to psychologists than for the consultations of private psychiatrists, the consultations of public psychiatrists in dedicated centers may contribute to reduce inequalities of access.

\section{Environmental influences on depression and related healthcare use}

Contrary to our expectations, we found no heterogeneity between neighborhoods regarding depressive symptoms. We only found a small inverse relationship between neighborhood median income (but not neighborhood social interactions) and depressive symptoms [39-41]. The observed relationship may be attributable, either to a causal effect of neighborhood poverty on the odds to develop depressive symptoms (accumulation of social disadvantage as a stressor) or to the fact that people with depressive symptoms may be disadvantaged on the job market and may have higher odds to move to socially deprived neighborhoods.

Regarding antidepressant consumption, we found almost no heterogeneity between neighborhoods, especially when individual socio-demographic variables were accounted for. 
Few studies [14, 15] have examined the relationship between residential environments and antidepressant consumption. In a Swedish study, one of the weakest associations reported between neighborhood deprivation and psychiatric prescription was for antidepressants [15].

However, using comparable administrative healthcare reimbursement data, a study conducted in another French city found more heterogeneity between neighborhoods than we did [14]. Potential explanations of this discrepancy include the different geographic location, the much larger (administrative-based) sample of this other study, and the fact that this previous study did not adjust for the depression status or various individual socio-demographic variables.

Regarding spatial accessibility to care, after adjustment for potential individual and neighborhood confounders, a high density of psychiatrists in a $1 \mathrm{~km}$ radius buffer around the residence was associated with greater odds to consult a psychiatrist. This relationship may capture a genuine effect of the density of psychiatrists, as the association was unchanged when we further controlled for the overall density of services in the residential buffer. A US study [42] showed that a poor spatial accessibility to the preferred provider was associated with a greater risk of not receiving a guideline-concordant treatment for depression, also pointing to an effect of spatial access to care.

However, we could not dismiss the hypothesis that the observed relationship is attributable to a demand of services induced by the psychiatrists themselves (some scholars have argued that in areas with a high density of physicians, physicians tend to encourage their patients to consult more regularly to compensate for their lower number of patients) [43, 44]. Another possible explanation for the observed relationship may be the selective location of practitioners, who may be more likely to settle down in neighborhoods where they anticipate a high demand of services. Unfortunately, we could not disentangle these three mechanisms with our data (spatial accessibility, induced demand, selective location of services). 
The relationship between neighborhood income and the consultation of psychiatrists was reduced in magnitude after adjustment for the density of psychiatrists. This finding is coherent with a study in Montreal [45] that showed a better spatial accessibility to mental health care in socially advantaged neighborhoods. The fact that the association between neighborhood income and the consultation of psychiatrists tended to persist after adjustment suggests that other aspects than the strict spatial accessibility to care may influence healthcare utilization behaviors, such as attitudes and values that may also be spatially patterned. Finally, our study could not take into account the local demand of mental health services in the area, which may affect the capacity to obtain an appointment with a physician.

\section{Conclusion}

Our study suggests that research on the relationships between the environments and depression and its treatment (i) should distinguish between the different facets of healthcare utilization (different types of providers, medications, etc.); and (ii) should consider the spatial accessibility to mental health services among the many environmental factors potentially involved in the onset and maintenance of depression. 


\section{Acknowledgments}

We are grateful to the National Institute for Prevention and Health Education (INPES, Institut National de Prévention et d'Education pour la Santé) (and Pierre Arwidson) for its continued support since the beginning of the study. We are grateful to Insee, the French National Institute of Statistics and Economic Studies, which provided support for the geocoding of the RECORD participants and allowed us to access to relevant geographical data (with special thanks to Pascale Breuil). We thank Geoconcept for allowing us to access to the Universal Geocoder software. We also thank CNAM-TS and the Caisse Primaire d'Assurance Maladie de Paris (CPAM-P, France) for helping make this study possible.

The RECORD project received support from the Institute for Public Health Research (IReSP, Institut de Recherche en Santé Publique); INPES (Prevention Program 2007; 2010-2011 financial support; and 2011-2013 financial support); the National Institute of Public Health Surveillance (InVS, Institut de Veille Sanitaire) (Territory and Health Program); the French Ministries of Research and Health (Epidemiologic Cohorts Grant 2008); the National Health Insurance Office for Salaried Workers (CNAM-TS, Caisse Nationale d'Assurance Maladie des Travailleurs Salariés); the Ile-de-France Regional Council (Conseil Régional d'Île-de-France, DIM SEnT and CODDIM); the National Research Agency (ANR, Agence Nationale de la Recherche) (Health-Environment Program 2005); the Ile-de-France Regional Health Agency (ARS, Agence Régionale de Santé) (in 2009; 2010; and 2011); the City of Paris (Ville de Paris); and the Ile-de-France Youth, Sports, and Social Cohesion Regional Direction (DRJSCS, Direction Régionale de la Jeunesse, des Sports et de la Cohésion Sociale). 


\section{References}

[1] Alonso J, Angermeyer MC, Bernert S, Bruffaerts R, Brugha TS, Bryson H, et al. Prevalence of mental disorders in Europe: results from the European Study of the Epidemiology of Mental Disorders (ESEMeD) project. Acta Psychiatr Scand Suppl 2004(420):21-7.

[2] Lorant V, Deliege D, Eaton W, Robert A, Philippot P, Ansseau M. Socioeconomic inequalities in depression: a meta-analysis. Am J Epidemiol 2003;157(2):98-112.

[3] Waraich P, Goldner EM, Somers JM, Hsu L. Prevalence and Incidence Studies of Mood Disorders: A Systematic Review of the Literature. Can J Psychiatry 2004;49(2):124-38.

[4] Mair C, Diez Roux AV, Galea S. Are neighbourhood characteristics associated with depressive symptoms? A review of evidence. J Epidemiol Community Health 2008;62(11):940-6.

[5] Paczkowski MM, Galea S. Sociodemographic characteristics of the neighborhood and depressive symptoms. Curr Opin Psychiatry 2010;23(4):337-41.

[6] Morin T. Classification des dépressifs selon leur type de recours aux soins. Etudes Et Resultats DREES 2007;577:8.

[7] Salines C, Cases E. Statistiques en psychiatrie en France : données de cadrage. Rev Fr Aff Soc 2004;1(1):181-204.

[8] Kovess-Masfety V, Saragoussi D, Sevilla-Dedieu C, Gilbert F, Suchocka A, Arveiller N, et al. What makes people decide who to turn to when faced with a mental health problem? Results from a French survey. BMC Public Health 2007;7:188.

[9] Briffault X, Sapinho D, Villamaux M, Kovess V. Factors associated with use of psychotherapy. Soc Psychiatry Psychiatr Epidemiol 2008;43(2):165-71. 
[10] Demyttenaere K, Bonnewyn A, Bruffaerts R, De Girolamo G, Gasquet I, Kovess V, et al. Clinical factors influencing the prescription of antidepressants and benzodiazepines: results from the European study of the epidemiology of mental disorders (ESEMeD). $\mathrm{J}$ Affect Disord 2008;110(1-2):84-93.

[11] Dezetter A, Briffault X, Alonso J, Angermeyer M, Bruffaerts R, De Girolamo G, et al. Factors Associated With Use of Psychiatrists and Nonpsychiatrist Providers by ESEMeD Respondents in Six European Countries. Psychiatric Services 2011;62(2):143-51.

[12] Dezetter A, Briffault X, Bruffaerts R, De Graaf R, Alonso J, König HH, et al. Use of general practitioners versus mental health professionals in six European countries: the decisive role of the organization of mental health-care systems. Social Psychiatry and Psychiatric Epidemiology 2013;48(1):137-49.

[13] Le Breton-Leourvillois G. Atlas de la démographie médicale en France. Situation au 1er janvier 2014: Conseil National de l'Ordre des Médecins; 2014.

[14] Bocquier A, Cortaredona S, Verdoux H, Sciortino V, Nauleau S, Verger P. Social Inequalities in new antidepressant treatment : a study at the individual and neighborhood levels. Ann Epidemiol 2013;23(3):99-105.

[15] Crump C, Sundquist K, Sundquist J, Winkleby MA. Neighborhood Deprivation and Psychiatric Medication Prescription: A Swedish National Multilevel Study. Ann Epidemiol 2011;21(4):231-7.

[16] Bruffaerts R, Posada-Villa J, Al-Hamzawi AO, Gureje O, Huang Y, Hu C, et al. Proportion of patients without mental disorders being treated in mental health services worldwide. The British Journal of Psychiatry 2014.

[17] Editors PM. The paradox of mental health: over-treatment and under-recognition. PLoS Med 2013;10(5):e1001456. 
[18] Ferrari AJ, Charlson FJ, Norman RE, Patten SB, Freedman G, Murray CJL, et al. Burden of Depressive Disorders by Country, Sex, Age, and Year: Findings from the Global Burden of Disease Study 2010. PLoS Med 2013;10(11):e1001547.

[19] Chaix B, Kestens Y, Bean K, Leal C, Karusisi N, Meghiref K, et al. Cohort Profile: Residential and non-residential environments, individual activity spaces and cardiovascular risk factors and diseases--The RECORD Cohort Study. Int J Epidemiol 2011.

[20] Havard S, Reich BJ, Bean K, Chaix B. Social inequalities in residential exposure to road traffic noise: an environmental justice analysis based on the RECORD Cohort Study. Occup Environ Med 2011;68(5):366-74.

[21] Chaix B, Bean K, Daniel M, Zenk SN, Kestens Y, Charreire H, et al. Associations of supermarket characteristics with weight status and body fat: a multilevel analysis of individuals within supermarkets (RECORD study). PLoS One 2012;7(4):e32908.

[22] Leal C, Bean K, Thomas F, Chaix B. Are associations between neighborhood socioeconomic characteristics and body mass index or waist circumference based on model extrapolations? Epidemiology 2011;22(5):694-703.

[23] Leal C, Bean K, Thomas F, Chaix B. Multicollinearity in associations between multiple environmental features and body weight and abdominal fat: using matching techniques to assess whether the associations are separable. Am J Epidemiol 2012;175(11):1152-62.

[24] Van Hulst A, Thomas F, Barnett TA, Kestens Y, Gauvin L, Pannier B, et al. A typology of neighborhoods and blood pressure in the RECORD Cohort Study. J Hypertens 2012;30(7):1336-46.

[25] Pichot P. Un questionnaire d'auto-évaluation de la symptomatologie dépressive, le questionnaire QD2. Forme abrégée QD2A. . Rev Psychol Appl 1984;34(4):323-40. 
[26] Chaix B, Chauvin P. [The contribution of multilevel models in contextual analysis in the field of social epidemiology: a review of literature]. Rev Epidemiol Sante Publique 2002;50(5):489-99.

[27] Merlo J, Chaix B, Ohlsson H, Beckman A, Johnell K, Hjerpe P, et al. A brief conceptual tutorial of multilevel analysis in social epidemiology: using measures of clustering in multilevel logistic regression to investigate contextual phenomena. J Epidemiol Community Health 2006;60(4):290-7.

[28] Greenland S, Robins JM, Pearl J. Confounding and Collapsibility in Causal Inference. Stat Sci 1999;14(1):29-46.

[29] Karlson KB, Holm A, Breen R. Comparing Regression Coefficients Between Samesample Nested Models Using Logit and Probit: A New Method. Sociol Methodol 2012;42(1):286-313.

[30] Chaix B, Billaudeau N, Thomas F, Havard S, Evans D, Kestens Y, et al. Neighborhood effects on health: correcting bias from neighborhood effects on participation. Epidemiology (Cambridge, Mass.) 2011;22(1):18-26.

[31] Kessler RC. Epidemiology of women and depression. J Affect Disord 2003;74(1):5-13.

[32] Seedat S, Scott KM, Angermeyer MC, Berglund P, Bromet EJ, Brugha TS, et al. Crossnational associations between gender and mental disorders in the WHO World Mental Health Surveys. Arch Gen Psychiatry 2009;66(7):785-95.

[33] Tsuang MT, Tohen M, Jones PB. Textbook of psychiatric epidemiology. 3rd edition. ed. Chichester, West Sussex: John Wiley \& Sons; 2011.

[34] Lorant V, Kampfl D, Seghers A, Deliege D, Closon MC, Ansseau M. Socio-economic differences in psychiatric in-patient care. Acta Psychiatr Scand 2003;107(3):170-7. 
[35] Andersen I, Thielen K, Nygaard E, Diderichsen F. Social inequality in the prevalence of depressive disorders. J Epidemiol Community Health 2009;63(7):575-81.

[36] Lorant V, Croux C, Weich S, Deliege D, Mackenbach J, Ansseau M. Depression and socio-economic risk factors: 7-year longitudinal population study. Br J Psychiatry 2007;190:293-8.

[37] Briffault X, Morvan Y, Rouillon F, Dardennes R, Lamboy B. [Factors associated with treatment adequacy of major depressive episodes in France]. Encephale 2010;36 Suppl 2:D59-72.

[38] Lostao L, Regidor E, Geyer S, Aiach P. Patient cost sharing and social inequalities in access to health care in three western European countries. Soc Sci Med 2007;65(2):36776.

[39] Henderson C, Diez Roux AV, Jacobs DR, Jr., Kiefe CI, West D, Williams DR. Neighbourhood characteristics, individual level socioeconomic factors, and depressive symptoms in young adults: the CARDIA study. J Epidemiol Community Health 2005;59(4):322-8.

[40] Kim D. Blues from the neighborhood? Neighborhood characteristics and depression. Epidemiol Rev 2008;30:101-7.

[41] Ross CE. Neighborhood Disadvantage and Adult Depression. J Health Soc Behav 2000;41(2):177-87.

[42] Fortney J, Rost K, Zhang M, Warren J. The impact of geographic accessibility on the intensity and quality of depression treatment. Med Care 1999;37(9):884-93.

[43] Rochaix L, Jacobzone S. [Empirical validation of the hypothesis that lead to the quest for the Graal?]. Cah Sociol Demogr Med 1997;37(3-4):183-203. 
[44] Trinquard S. Demande induite par l'offre ambulatoire : un survol de la littérature théorique et empirique. LASER Working Papers 2006.

[45] Ngamini Ngui A, Apparicio P. [Potential accessibility to mental health services in Montreal: a geographical information system approach]. Rev Epidemiol Sante Publique 2011;59(6):369-78. 


\section{Table 1}

Variations between residential areas in the odds of depressive symptoms and healthcare utilization, as assessed from 2-level multilevel models adjusted for age and gender (separate models with municipalities, TRIRIS neighborhoods, and IRIS neighborhoods as level-2 units)

\begin{tabular}{lccc}
\hline & $\begin{array}{c}\text { TRIRIS } \\
(\mathrm{N}=112)\end{array}$ & $\begin{array}{c}\text { IRIS } \\
\text { neighborhoods } \\
(\mathrm{N}=662)\end{array}$ & $\begin{array}{c}\text { neighborhoods } \\
(\mathrm{N}=1914)\end{array}$ \\
\hline Depressive symptoms (n=7290) & & & \\
$\quad$ Variance (SE) & $0.117(0.058)$ & $0.064(0.065)$ & $0.252(0.128)$ \\
$\quad$ Median odds ratio & 1.385 & 1.274 & 1.615 \\
P value & 0.004 & 0.146 & 0.014 \\
Antidepressant consumption (n=7226) & & & \\
$\quad$ Variance (SE) & $0.021(0.027)$ & $0.032(0.053)$ & $0.074(0.090)$ \\
Median odds ratio & 1.148 & 1.186 & 1.296 \\
P value & 0.136 & 0.266 & 0.198 \\
Psychiatrist Consultation (n=7226) & & & \\
$\quad$ Variance (SE) & $0.144(0.081)$ & $0.231(0.114)$ & $0.387(0.177)$ \\
Median odds ratio & 1.437 & 1.581 & 1.810 \\
P value & $<0.001$ & 0.012 & 0.008 \\
\hline
\end{tabular}




\section{Table 2}

Associations of individual sociodemographic variables and neighborhood median income with the odds of depressive symptoms. assessed from a multilevel logistic model with participants nested within IRIS neighborhoods

\section{Depressive Symptoms}

Individual-level model

Model 1

$\mathrm{n}=7213, \mathrm{~N}=1907$

OR $(95 \% \mathrm{CI})$

\section{Gender}

Men

Women

Age

$30-44$

45-59

60-79

Marital Status

Couple

Alone

Educational level

Upper tertiary

Higher secondary and lower tertiary

Primary education and lower secondary

No education

Employment Status

Employed

Unemployed

Retired

Financial strain

No

Yes

Median neighborhood income

Q1

Q2

Q3

Q4

Random effect

IRIS level variance (SE)

Median odds ratio

$P$ value for the variance
$1.6(1.3-1.9)$

1

$1.0(0.8-1.3)$

$1.1(0.9-1.3)$

$0.6(0.4-1.0)$

1

$2.0(1.7-2.5)$

1

$1.4(1.1-1.7)$

1.3 (1.0-1.7)

$1.4(1.1-1.8)$

1.3 (1.0-1.7)

$1.9(1.4-2.6)$

$1.7(1.2-2.3)$

1

1

1.5 (1.2-1.9)

$1.5(1.2-1.9)$

$1.9(1.4-2.7)$

1.9 (1.3-2.7)

1

$3.0(2.4-3.7)$

$2.8(2.3-3.5)$

1

0.8 (0.6-1.0)

0.7 (0.5-0.9)

0.8 (0.6-1.0)

$0.150(0.134)$

1.44

$0.131(0.133)$

0.115

0.149 
Table 3

Associations between individual sociodemographic variables and the odds of consultation of psychiatrists or the odds of antidepressant consumption, assessed from multilevel logistic models with participants nested within IRIS neighborhoods

\begin{tabular}{|c|c|c|}
\hline & $\begin{array}{c}\text { Consultation } \\
\text { of psychiatrists }\end{array}$ & $\begin{array}{c}\text { Antidepressant } \\
\text { consumption }\end{array}$ \\
\hline & $\begin{array}{c}\text { Model } 1 \\
\mathrm{n}=6984, \mathrm{~N}=1895 \\
\text { OR }(95 \% \mathrm{CI})\end{array}$ & $\begin{array}{c}\text { Model 1 } \\
\mathrm{n}=6813, \mathrm{~N}=1886 \\
\text { OR }(95 \% \mathrm{CI})\end{array}$ \\
\hline \multicolumn{3}{|l|}{ Gender } \\
\hline Men & 1 & 1 \\
\hline Women & $1.3(1.0-1.6)$ & $1.9(1.6-2.3)$ \\
\hline \multicolumn{3}{|l|}{ Age } \\
\hline $30-44$ & 1 & 1 \\
\hline $45-59$ & $1.1(0.8-1.4)$ & $1.3(1.1-1.6)$ \\
\hline $60-79$ & $0.7(0.5-0.9)$ & $1.2(0.9-1.5)$ \\
\hline \multicolumn{3}{|l|}{ Country of Birth } \\
\hline Other than France & & 1 \\
\hline France & $2.0(1.5-2.8)$ & $1.4(1.2-1.7)$ \\
\hline \multicolumn{3}{|l|}{ Parents' educational level } \\
\hline University level & 1 & 1 \\
\hline Secondary level & $0.7(0.5-0.9)$ & $0.9(0.7-1.1)$ \\
\hline Primary level or less & $0.6(0.5-0.9)$ & $0.7(0.6-0.9)$ \\
\hline \multicolumn{3}{|l|}{ Marital status } \\
\hline Couple & 1 & 1 \\
\hline Alone & $1.6(1.3-2.1)$ & $1.3(1.1-1.6)$ \\
\hline \multicolumn{3}{|l|}{ Educational level } \\
\hline Upper tertiary & 1 & 1 \\
\hline Higher secondary and lower tertiary & $0.7(0.5-0.9)$ & $1.5(1.2-1.8)$ \\
\hline Primary education and lower secondary & $0.6(0.4-0.9)$ & $1.4(1.1-1.8)$ \\
\hline No education & $0.4(0.2-0.8)$ & $1.4(1.0-2.0)$ \\
\hline \multicolumn{3}{|l|}{ Household income per consumption unit } \\
\hline$>2201 €$ & 1 & \\
\hline$<2201 €$ & $1.2(0.9-1.7)$ & \\
\hline$<1376 €$ & $0.9(0.6-1.3)$ & \\
\hline$<833 €$ & $1.5(1.0-2.1)$ & \\
\hline \multicolumn{3}{|l|}{ Health Insurance } \\
\hline Private complementary insurance & & 1 \\
\hline Public complementary insurance & & $1.3(1.0-1.8)$ \\
\hline No complementary insurance & & $0.8(0.6-1.1)$ \\
\hline \multicolumn{3}{|l|}{ Depressive status } \\
\hline No & 1 & 1 \\
\hline Yes & $3.5(2.6-4.9)$ & $6.0(4.8-7.4)$ \\
\hline \multicolumn{3}{|l|}{ Random effect } \\
\hline IRIS level variance (SE) & $0.346(0.180)$ & $0.022(0.100)$ \\
\hline Median odds ratio & 1.753 & 1.150 \\
\hline $\mathrm{P}$ value for the variance & 0.017 & 0.4138 \\
\hline
\end{tabular}


Table 4

Associations between neighborhood variables and the odds of consultation of psychiatrists, assessed from multilevel logistic models adjusted for individual sociodemographic characteristics with participants nested within IRIS neighborhoods

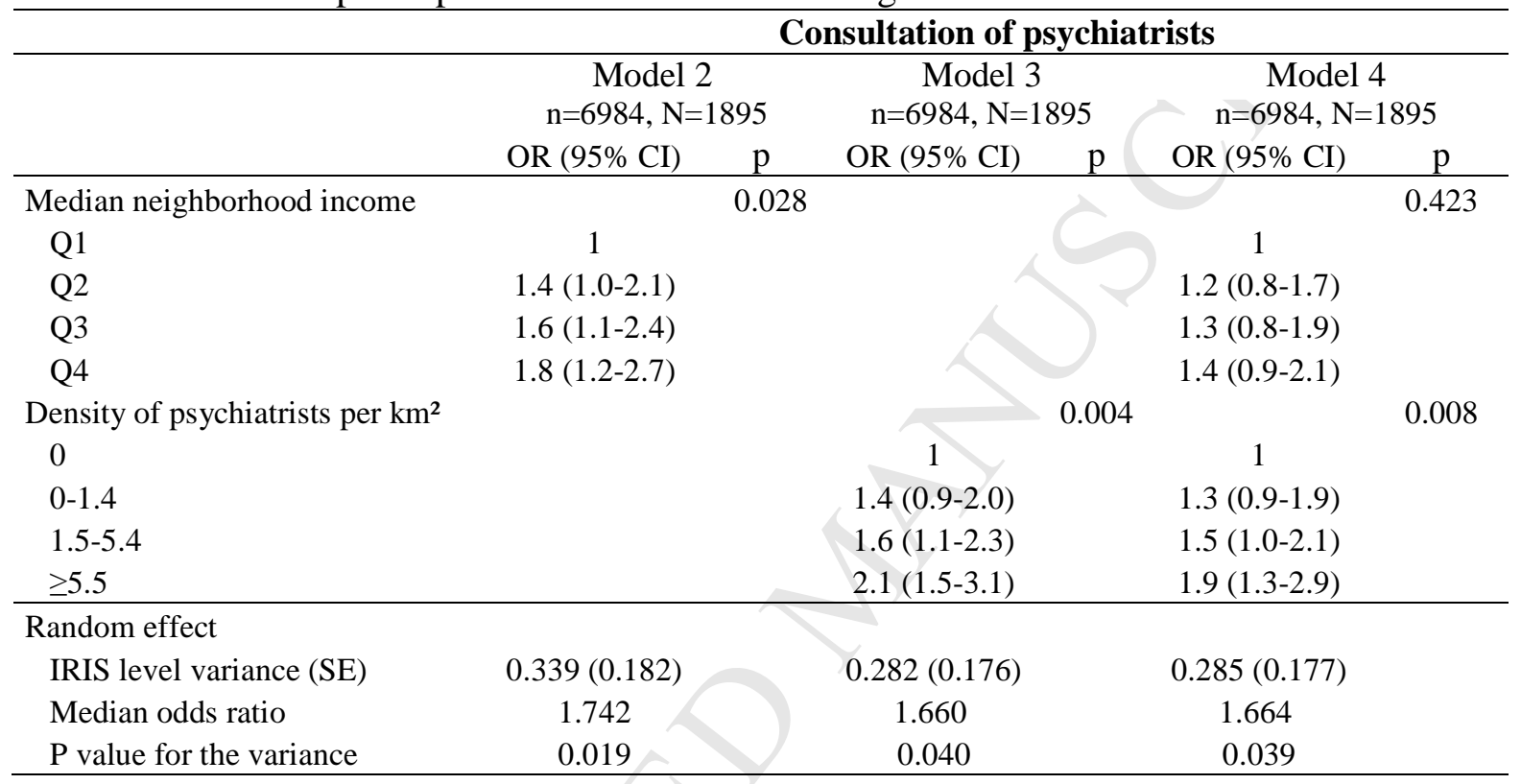


Table 5: Rate of use of antidepressants and consultation of psychiatrists in the whole RECORD population and among participants with depressive symptoms

\begin{tabular}{|c|c|c|c|c|c|c|c|c|}
\hline & \multicolumn{4}{|c|}{ Total Record sample (n=7226) } & \multicolumn{4}{|c|}{ Depressive population (n=566) } \\
\hline & \multicolumn{2}{|c|}{$\begin{array}{l}\text { Consumption of } \\
\text { antidepressants }\end{array}$} & \multicolumn{2}{|c|}{$\begin{array}{l}\text { Psychiatric } \\
\text { consultation }\end{array}$} & \multicolumn{2}{|c|}{$\begin{array}{l}\text { Consumption of } \\
\text { antidepressants }\end{array}$} & \multicolumn{2}{|c|}{$\begin{array}{l}\text { Psychiatric } \\
\text { consultation }\end{array}$} \\
\hline & $\%(\mathrm{n})$ & $\mathrm{P}$ & $\%(\mathrm{n})$ & $\mathrm{p}$ & $\%(\mathrm{n})$ & $\mathrm{p}$ & $\%(\mathrm{n})$ & $\mathrm{p}$ \\
\hline Total & $10.0 \%(719)$ & & $4.7 \%(33$ & & $36.0 \%(202)$ & & $12.1 \%(68)$ & \\
\hline Gender & & $<0.001$ & & 0.019 & & 0.084 & & 0.037 \\
\hline Men & $7.2 \%(341)$ & & 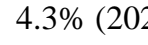 & & $32.5 \%(91)$ & & $15.0 \%(42)$ & \\
\hline Women & $15.2 \%(378)$ & & $5.5 \%(13)$ & & $39.5 \%(111)$ & & $9.3 \%(26)$ & \\
\hline Age (years)* & & 0.088 & & 0.004 & V & 0.268 & & 0.299 \\
\hline $30-44$ & $8.9 \%(229)$ & & $5.2 \%(13$ & & $34.4 \%(75)$ & & $13.3 \%(29)$ & \\
\hline $45-59$ & $10.7 \%(321)$ & & $5.1 \%(15$ & & $35.1 \%(85)$ & & $12.4 \%(30)$ & \\
\hline $60-79$ & $10.3 \%(169)$ & & $3.1 \%(51$ & & $41.6 \%(42)$ & & $8.9 \%(9)$ & \\
\hline Educational level* & & $<0.001$ & & $<0.001$ & & 0.284 & & 0.004 \\
\hline No education & $11.5 \%(65)$ & & $2.3 \%(13$ & & $29.9 \%(23)$ & & $3.9 \%(3)$ & \\
\hline Primary education and lower secondary & $11.5 \%(203)$ & & $3.9 \%(68$ & & $34.3 \%(59)$ & & $10.5 \%(18)$ & \\
\hline Higher secondary and lower tertiary & $11.6 \%(245)$ & & $4.3 \%(92$ & & $39.8 \%(70)$ & & $10.8 \%(19)$ & \\
\hline Upper tertiary & $7.2 \%(197)$ & & $6.0 \%(16$ & & $35.9 \%(46)$ & & $21.1 \%(27)$ & \\
\hline Income level & & & & 0.437 & & 0.888 & & 0.008 \\
\hline$<833 €$ & $13.1 \%(247)$ & & $4.9 \%(93$ & & $35.5 \%(89)$ & & $9.2 \%(23)$ & \\
\hline$<1376 €$ & $9.5 \%(165)$ & & $3.6 \%(63$ & & $36.0 \%(45)$ & & $9.6 \%(12)$ & \\
\hline$<2201 €$ & $8.6 \%$ (139) & & $5.4 \%(87$ & & $39.5 \%(34)$ & & $18.6 \%(16)$ & \\
\hline$>2201 €$ & $8.6 \%(166)$ & & $5.0 \%(96$ & & $34.7 \%(34)$ & & $17.4 \%(17)$ & \\
\hline
\end{tabular}

*P value from a Chi2 trend test 